\title{
Pair-cluster theory for the dielectric constant of composite media
}

\author{
Ping Sheng \\ Theoretical Sciences Group, Corporate Research Science Laboratories, Exxon Research and Engineering Company, \\ P. O. Box 45, Linden, New Jersey 07036 \\ (Received 10 July 1980)
}

By considering the embedding of nearest-neighbor grains of a random composite in an "effective medium," a paircluster theory for the dielectric constant of composites is formulated in which the interactions between pairs of nearest-neighbor grains are explicitly taken into account. Compared to the well-known effective-medium theory, the new theory displays similar dc behavior but predicts different optical dielectric constants. A comparison of the two theories shows in particular that the absence of optical dielectric anomaly in the effective-medium theory is attributable to the neglect of nearest-neighbor interactions. Differences between the optical characteristics of the Maxwell-Garnett theory and the pair-cluster theory are also pointed out and discussed in relation to the underlying microstructures.

The effective-medium theory ${ }^{1,2}$ (EMT) and the Maxwell-Garnett theory ${ }^{3}$ (MGT) are the two most widely used mean-field-type theories for the calculation of dielectric constants of composite media. Both theories are derived on the assumption that the composite material consists of grains which are much smaller than the wavelength of the probing electromagnetic radiation. However, they differ in the treatment of the two components in a composite system. Whereas in the EMT the two components are treated in an equivalent manner, in the MGT the grains of one component are taken to be embedded in the matrix of the other component. The different microstructures implicitly implied by the two theories are schematically illustrated in Fig. 1. In a recent paper, ${ }^{4}$ Gittleman et al. have pointed out that the EMT and the MGT predict grossly different optical dielectric constants. In particular, the EMT does not produce the optical dielectric anomaly observed in granular metal films, ${ }^{5,6}$ while the MGT does predict the existence of the anomaly. In view of the demonstrated link ${ }^{7}$ between the optical and dc transport properties of granular metals and its underlying microstructure, ${ }^{8,9}$ the disagreement between the two theories raises an interesting question: Is the absence of the optical dielectric anomaly in the EMT an inherent consequence of its microstructure or simply a reflection of the approximation used in the derivation of EMT? It is the purpose of this note to answer the above question by constructing an alternative "pair cluster" form of the EMT in which the interactions between pairs of nearest-neighbor grains are explicitly taken into account. For metal-insulator composites, the new theory displays similar dc behavior as the EMT (see Fig. 3). However, at optical frequencies the pair-cluster theory is shown to exhibit dielectric anomaly. Since the microstructure treated by the pair-cluster theory is implicitly the same as that for the EMT, the difference between the two theories clearly points to the neglect of nearest-neighbor interactions as the culprit for the absence of dielectric anomaly in the EMT.

Consider a random composite of two components, $\alpha$ and $\beta$, schematically depicted in Fig. 1 . In the EMT, grains of $\alpha$ and $\beta$ are assumed to be embedded in a uniform effective medium of dielectric constant $\bar{\epsilon}$. When an electric field $E$ is applied, the field inside each grain, $E_{\alpha(\beta)}$, can be easily calculated in the case of spherical grain geometry:

$$
E_{\alpha(\beta)}=\frac{3 \bar{\epsilon}}{\epsilon_{\alpha(\beta)}+2 \bar{\epsilon}} E .
$$

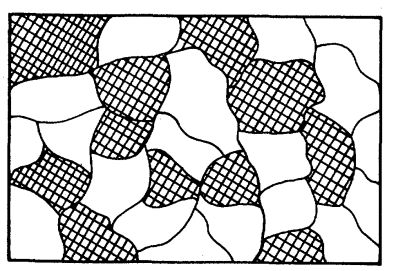

(a)

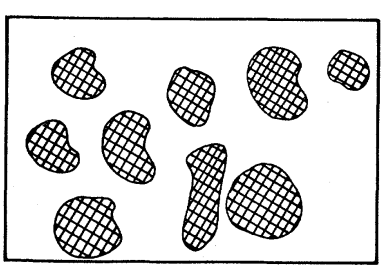

(b)

FIG. 1. (a) Microstructure treated by the effectivemedium theory. (b) Microstructure treated by the Maxwell-Garnett theory. 
The deviation of $E_{\alpha(\beta)}$ from the uniform field value $E$ is simply given by $\Delta E_{\alpha(\beta)}=E_{\alpha(\beta)}-E$. By imposing the effective medium condition that the deviations should average to zero, we obtain the EMT equation:

$$
p \frac{\epsilon_{\alpha}-\bar{\epsilon}}{\epsilon_{\alpha}+2 \bar{\epsilon}}+(1-p) \frac{\epsilon_{\beta}-\bar{\epsilon}}{\epsilon_{\beta}+2 \bar{\epsilon}}=0,
$$

where $p$ is the volume fraction of component $\alpha$. In the derivation of Eq. (2) it is important to note that the only interfaces explicitly considered are those between the individual grains of $\alpha$ (or $\beta$ ) and the effective medium. Therefore, if there are effects intrinsically associated with the inter faces between the $\alpha$ and $\beta$ components, they are not expected to be adequately accounted for by the EMT. In order to overcome this deficiency, let us consider the alternative scheme of embedding a pair of nearest-neighbor grains in a uniform effective medium. In the same spirit as the EMT, we will approximate the geometry of the twograin pair by a sphere in which each half can be either component $\alpha$ or $\beta$. The general problem is then the solution of the electrostatic problem shown in Fig. 2(a). However, it is clear that the solution of the general case is obtainable as the superposition of the two special cases illustrated in Fig. 2(b), where the applied electric field is either parallel or perpendicular to the plane separating the two components. In the perpendicular case, the electric potential inside and outside the sphere can be written in the expansions

$V_{\text {in }}=\sum_{l=0}^{\infty} r^{2 l+1} \epsilon(\theta) A_{2 l+1}^{V} P_{2 l+1}(\cos \theta)+r^{2 t} B_{2 l}^{V} P_{2 l}(\cos \theta)$,

$V_{\text {out }}=-E r \cos \theta+\sum_{i=0}^{\infty} r^{-(l+1)} C_{i}^{V} P_{l}(\cos \theta)$,

where $\theta$ is the angle measured from the $z$ direction, $r$ is the radial distance, $P_{l}(\cos \theta)$ stands for $l$ th Legendre polynomial, and $\epsilon(\theta)$ is equal to the dielectric constant of the upper hemisphere for $0 \leqslant \theta \leqslant \pi / 2$ and equal to the dielectric constant of the lower hemisphere for $\pi / 2 \leqslant \theta \leqslant \pi$. Similar expansions in the parallel case are given by

$$
\begin{aligned}
V_{1 \mathrm{n}}= & \sum_{l=0}^{\infty} r^{2 l+1} A_{2 l+1}^{H} P_{2 l-1}^{(1)}(\cos \theta) \cos \phi \\
& +r^{2 t} B_{2 l}^{H} \epsilon(\theta) P_{2 l}^{(1)}(\cos \theta) \cos \phi \\
V_{\text {out }}= & -E r \sin \theta \cos \phi \\
+ & \sum_{l=0}^{\infty} r^{-(t+1)} C_{l}^{H} P_{l}^{(1)}(\cos \theta) \cos \phi
\end{aligned}
$$

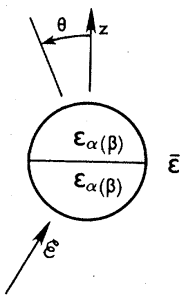

(a)
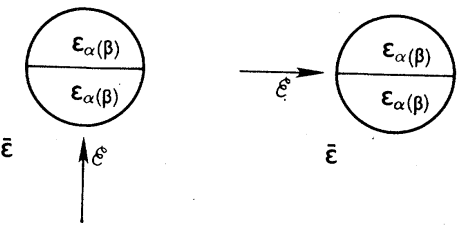

(b)

FIG. 2. Solution to the general electrostatic problem shown in (a), where a sphere with distinct dielectric constant in each half is polarized by a uniform field applied at arbitrary angle, can be decomposed into two simpler problems shown in (b), where the applied field is either perpendicular or parallel to the plane separating the two halves.

where $\phi$ is the azimuthal angle and $P_{l}^{(1)}(\cos \theta)$ is the associated Legendre function of the first order. By imposing the boundary conditions

$$
\begin{aligned}
& V_{\text {in }}=V_{\text {out }} \text { at } r=1, \\
& \epsilon(\theta) \partial_{r} V_{\text {in }}=\bar{\epsilon} \partial_{r} V_{\text {out }} \text { at } r=1,
\end{aligned}
$$

and multiplying through by $P_{l}(\cos \theta)\left[\right.$ or $P_{l}^{(1)}(\cos \theta)$ $\cos \phi$ ], we can easily integrate both sides of Eqs. (7) and (8) by utilizing the orthogonality properties of Legendre polynomials and their tabulated integrals. ${ }^{10}$ The resulting series contain no dependence on either $\theta$ or $\phi$ and form sets of linear simultaneous equations from which the coefficients $A_{l}^{V^{(H)}}, B_{l}^{V^{(H)}}$, and $C_{l}^{V^{(H)}}$ are explicitly determined. In terms of $A_{l}^{V^{(H)}}$ and $B_{l}^{V^{(H)}}$, one can easily express the average field deviation $\Delta \overrightarrow{\mathrm{E}}$ (from the uniform applied field $E$ ) within any given sphere. The effective-medium condition, that the average of $\Delta \overrightarrow{\mathrm{E}}$ over all orientations and all two-hemisphere combinations should equal zero, then yields

$$
\begin{array}{r}
p^{2} \frac{\bar{\epsilon}-\epsilon_{1}}{2 \bar{\epsilon}+\epsilon_{1}}+(1-p)^{2} \frac{\bar{\epsilon}-\epsilon_{2}}{2 \bar{\epsilon}+\epsilon_{2}}+\frac{4}{9} p(1-p) \gamma_{1}^{H}\left(1-\frac{\epsilon_{1}+\epsilon_{2}}{2 \bar{\epsilon}}\right) \\
+\frac{2}{9} p(1-p) \gamma_{1}^{V}\left(\frac{\epsilon_{1}+\epsilon_{2}}{2}-\frac{\epsilon_{1} \epsilon_{2}}{\bar{\epsilon}}\right)=0 .
\end{array}
$$

Here $\gamma_{1}^{H}$ and $\gamma_{1}^{V}$ are obtained from the solutions of the following sets of linear equations: 


$$
\begin{aligned}
2 \bar{\epsilon} \delta_{1 l}= & \frac{2 l(2 l-1)}{4 l-1}\left(\frac{2 l-1}{2}\left(\epsilon_{1}+\epsilon_{2}\right)+2 l \bar{\epsilon}\right) \gamma_{2 l-1}^{H} \\
& +l \bar{\epsilon}\left(\epsilon_{1}-\epsilon_{2}\right)^{2} \sum_{m=1}^{\infty}(2 m-1) K_{l m} \gamma_{2 m-1}^{H}, \\
\bar{\epsilon} \delta_{1 l}= & \frac{\left[l \bar{\epsilon}\left(\epsilon_{1}+\epsilon_{2}\right)+(2 l-1) \epsilon_{1} \epsilon_{2}\right]}{4 l-1} \gamma_{2 l-1}^{V} \\
& +\frac{\left(\epsilon_{1}-\epsilon_{2}\right)^{2} \bar{\epsilon}}{2} \sum_{m=1}^{\infty} H_{l m} \gamma_{2 m-1}^{V},
\end{aligned}
$$

where

$$
K_{l m}=\sum_{n=1}^{\infty} \frac{n(2 n+1)(4 n+1) J_{n t} J_{n m}}{\left[2 n \epsilon_{1} \epsilon_{2}+\left(n+\frac{1}{2}\right) \bar{\epsilon}\left(\epsilon_{1}+\epsilon_{2}\right)\right]}
$$

$$
\begin{gathered}
H_{l m}=\sum_{n=1}^{\infty} \frac{n(2 n+1)(4 n+1) J_{n l} J_{n m}}{\left[n\left(\epsilon_{1}+\epsilon_{2}\right)+(2 n+1) \bar{\epsilon}\right]}, \\
J_{m l}=\frac{(-1)^{m+l} 2 m !(2 l-1) !}{4^{m+l-1} 2[m !(l-1) !]^{2}(m+l)(2 m-2 l+1)} .
\end{gathered}
$$

By truncating the infinite series in Eqs. (10) and (11) to $N$ terms and solving the resulting equations numerically for various $N$ values, it was found that results accurate to better than $1 \%$ are achieved by retaining only the leading terms in the series. In that case Eqs. (9)-(11) can be combined to yield

$p^{2} \frac{\bar{\epsilon}-\epsilon_{1}}{2 \bar{\epsilon}+\epsilon_{1}}+(1-p)^{2} \frac{\bar{\epsilon}-\epsilon_{2}}{2 \bar{\epsilon}+\epsilon_{2}}+\frac{4}{9} p(1-p) \frac{2 \bar{\epsilon}-\epsilon_{1}-\epsilon_{2}}{K \bar{\epsilon}\left(\epsilon_{1}-\epsilon_{2}\right)^{2}+\left(\epsilon_{1}+\epsilon_{2}+4 \bar{\epsilon}\right) / 3}$

$$
+\frac{2}{9} p(1-p) \frac{\bar{\epsilon}\left(\epsilon_{1}+\epsilon_{2}\right)-2 \epsilon_{1} \epsilon_{2}}{H \bar{\epsilon}\left(\epsilon_{1}-\epsilon_{2}\right)^{2}+2\left(\epsilon_{1} \epsilon_{2}+\bar{\epsilon} \epsilon_{1}+\bar{\epsilon} \epsilon_{2}\right) / 3}=0,
$$

with

$$
\begin{aligned}
& H=\frac{1}{4} \sum_{m=1}^{\infty} \frac{I_{m}}{\left[m\left(\epsilon_{1}+\epsilon_{2}\right)+(2 m+1) \bar{\epsilon}\right]}, \\
& K=\frac{1}{4} \sum_{m=1}^{\infty} \frac{I_{m}}{\left[2 m \epsilon_{1} \epsilon_{2}+(n+1 / 2)\left(\epsilon_{1}+\epsilon_{2}\right) \bar{\epsilon}\right]}, \\
& I_{m}=\frac{m(4 m+1)[(2 m+1) !]^{2}}{4^{2 m}(m !)^{4}(2 m-1)^{2}(m+1)^{2}(2 m+1)} .
\end{aligned}
$$

The dc percolation transport property of the pair-cluster model is obtained from Eqs. (15)(18) by using $\epsilon_{1}=1$ (or $i$ ) and $\epsilon_{2}=0$. The result is

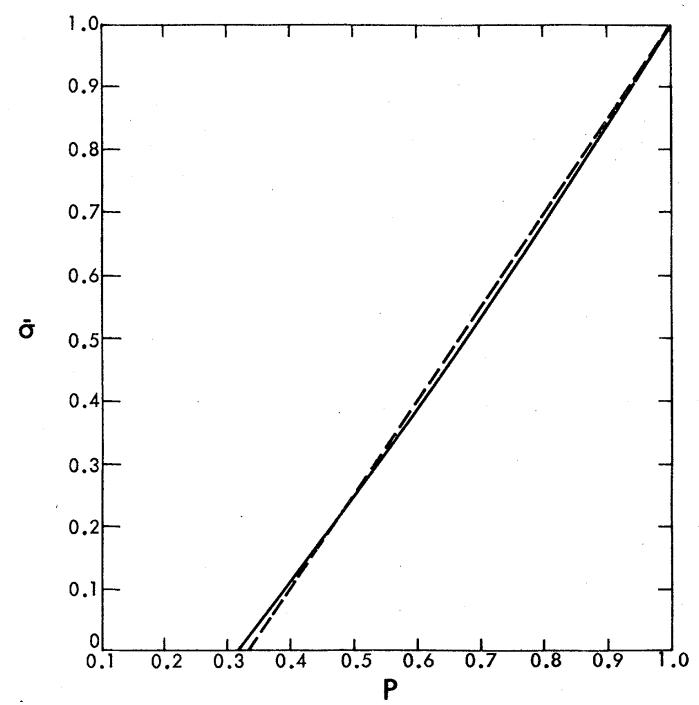

FIG. 3. Effective conductivity $\bar{\sigma}$ as a function of composition $p$ for the pair-cluster theory (-) and the effective-medium theory (---). shown in Fig. 3. It is seen that although the percolation threshold, $p_{c}=0.31$, differs from the EMT value of $p_{c}=0.33$, the overall variation of the effective conductivity $\bar{\sigma}$ as a function of $p$ is very similar to the EMT. In Fig. 4 we show the

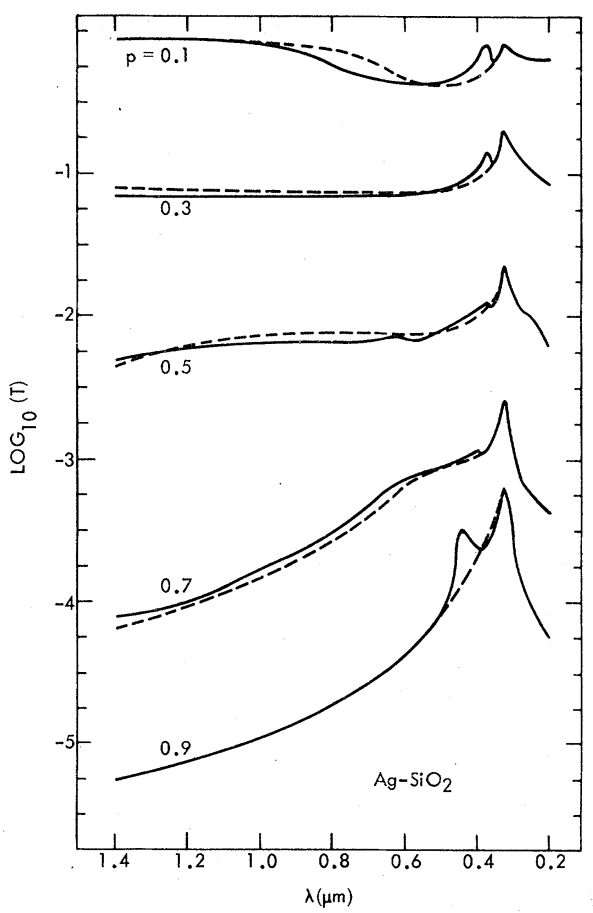

FIG. 4. Calculated optical transmission as a function of light wavelength for a series of $500-\AA$-thick $\mathrm{Ag}-\mathrm{SiO}_{2}$ composite films. The dashed line denotes the effectivemedium theory. The values of composition $p$ are labeled in the figure. For clarity, the pairs of curves are displaced vertically with respect to one another. 


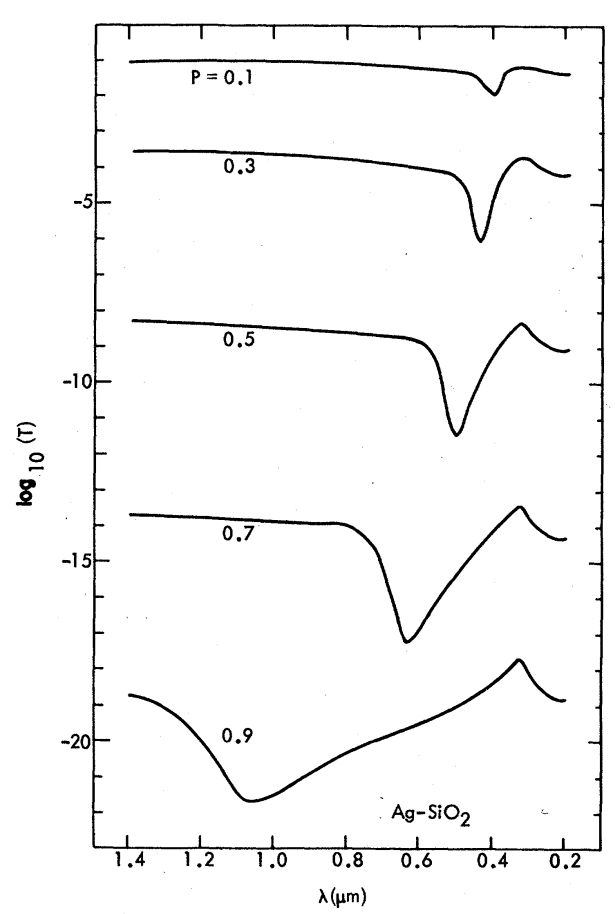

FIG. 5. Optical transmission for a series of $500-\AA-$ thick $\mathrm{Ag}-\mathrm{SiO}_{2}$ composite films calculated by the MaxwellGarnett theory.

calculated optical transmission spectrum for a series of $500-\AA$-thick $\mathrm{Ag}-\mathrm{SiO}_{2}$ films. The optical dielectric constant of the composite is evaluated by using both the pair-cluster theory (solid line) and the EMT (dashed line). For $\epsilon_{1}$ I used the experimentally determined constants ${ }^{11}$ of $\mathrm{Ag}$, modified to take into account the decrease of conduction-electron relaxation time $\tau$ due to small-particle microstructure. ${ }^{5}$ The value of $\tau$ used in the calculation is $2.5 \times 10^{-15} \mathrm{sec}$, cor responding to a particle size of $50 \AA$. For $\epsilon_{2}$ the constant value of 2.2 was employed. It is easily seen that the pair-cluster theory exhibits an absorption peak, the dielectric anomaly, which the EMT does not have. In addition, the pair-cluster theory also shows an extra transmission peak. Since the pair-cluster model differs from the EMT primarily in the treatment of nearest-neighbor interaction, the origin of the dielectric anomaly and the extra transmission peak can clearly be identified as local in nature.

Compared to the MGT, the location of the absorption peak in the pair-cluster theory and its variation with composition $p$ are very different, as seen from Figs. 4 and 5. Whereas in the MGT the absorption peak shifts down in frequency and becomes more pronounced as $p$ approaches 1 , in the pair-cluster model the dielectric anomaly stays at a relatively fixed frequency and disappears in the range $0.7>p>0.4$. Such dis similarities are traceable to the different underlying microstructures in the two cases. Since the shift of absorption peak (toward lower frequencies) in the MGT is associated with decreasing (relative) thickness of the oxide coating on metallic particles, it is understandable that the same peak should not vary its position in the pair-cluster model, because in that case the oxide and metallic grains have a minimum size which does not vary with the composition. On the other hand, the disappearance of the absorption peak for $0.7>p>0.4$ can be ascribed to the occurrence of a matrix inversion within that composition region, and in contrast to the MGT, at $p>0.7$ the absorption peak of the pair-cluster theory is associated with the resonance caused by the insulating inclusions in the metal continuum. ${ }^{12}$ It is interesting to note that the matrix inversion in the pair-cluster theory and the EMT can actually be discerned by the transition in the infrared behavior from a frequency-independent transmission (characteristic of $\mathrm{SiO}_{2}$ ) to a transmission which increases with frequency (characteristic of $\mathrm{Ag}$ ).

In summary, since the pair-cluster theory and the EMT represent two different treatments for the same composite microstructure, the comparison of the two theories has revealed that the optical dielectric constant is sensitive to the short-range interactions while the dc transport behavior is relatively impervious to approximations on the local environment of a grain. Further comparison between the pair-cluster theory and the Maxwell-Garnett theory has illustrated the effect of different microstructures on the optical properties of composites.

The author wishes to thank R. Cohen for helpful discussions.
${ }^{1}$ D. A. G. Bruggeman, Ann. Phys. (Leipzig) 24, 636 (1935).

${ }^{2}$ R. Landauer, J. Appl. Phys. 23, 779 (1952).

${ }^{3}$ J. C. M. Garnett, Philos. Trans. R. Soc. London 203 , 385 (1904).

${ }^{4}$ J. I. Gittleman and B. Abeles, Phys. Rev. B 15, 3273 (1977).
${ }^{5}$ R. W. Cohen, G. D. Cody, M. D. Coretts, and B. Abeles, Phys. Rev. B 8,3689 (1973).

${ }^{6}$ D. Stroud, Phys. Rev. B 12, 3368 (1975).

${ }^{7}$ P. Sheng, Phys. Rev. Lett. 45, 60 (1980).

${ }^{8}$ The microstructure of granular composites cannot be realistically accounted for by either the EMT or the MGT (see Ref. 7). 
${ }^{9}$ R. Mc Phedran and D. O. Mc Kenzie, Proc. R. Soc. London, Ser. A 359, 45 (1978); D. Bergman, J. Phys. C 12, 4947 (1979).

${ }^{10} \mathrm{M}$. Abramowitz and I. Stegun, Handbook of Mathemati- cal Functions (Dover, New York, 1965), Chap. 8. ${ }^{11}$ P. B. Johnson and R. W. Christy, Phys. Rev. B 6 , 4370 (1972).

${ }^{12}$ F. L. Galeener, Phys. Rev. 27, 421 (1971). 